\title{
A framework for the observer design for networked control systems
}

\author{
Romain Postoyan and Dragan Nešić
}

\begin{abstract}
This paper proposes a framework for the observer design for networked control systems (NCS) affected by disturbances, via an emulation-like approach. The proposed model formulation allows us to consider various static and dynamic time-scheduling protocols, in-network processing implementations and encompasses sampled-data systems as a particular case. Provided that the continuous-time observer is robust to the measurement errors (in an appropriate sense) we derive bounds on the maximum allowable transmission interval (MATI) that ensure the convergence of observation errors under network-induced communication constraints. The stability analysis is trajectory-based and utilizes small-gain arguments. A number of observers can be combined and used within our approach to obtain estimators for NCS.
\end{abstract}

\section{INTRODUCTION}

Networked control systems (NCS) are systems in which the sensors and the actuators are spatially distributed and communicate with the control structure via a network. At each transmission instant, only a subset of sensors and/or actuators, collocated into a node, is chosen to transmit their data over the communication channel according to a scheduling rule called protocol. The growing interest for NCSs is motivated by the fact that they have lower costs, easier maintenance and installation, greater flexibility as well as lower weight and volume in comparison to the classical control systems. On the other hand, the communication constraints induced by the use of a serial channel cannot be ignored and require novel observation and control design methods.

R. Postoyan is with the Centre de Recherche en Automatique de Nancy, UMR 7039, Nancy Université, CNRS, 2 avenue de la forêt de Haye, 54516 Vandoeuvre-lès-Nancy, France. E-mail: romain.postoyan@cran.uhp-nancy . fr. Supported by the French Ministère de l'Education Nationale de la Recherche et de la Technologie (MENRT) and the LSS-CNRS, SUPELEC.

D. Nešić is with the Department of Electrical and Electronic Engineering, University of Melbourne, Parkville 3010, Victoria, Australia. E-mail: d.nesic@ee.unimelb.edu.au. Supported by the Australian Research Council under the Future Fellow and Discovery Project programs. 
In this paper, we focus on the design of a centralized observer that receives data from sensors through a shared communication medium. Related available studies in the literature have addressed this problem for particular classes of systems or protocols. In [3], a methodology for the mutual design of weighted dynamic protocols and observer gains is developed for linear systems using matrix inequalities. The existence of a round-robin protocol that preserves the observability of linear discrete-time NCS is ensured in [16] under mild conditions. Using linear time-varying periodic systems analysis tools, a linear observer is then derived for such a protocol. The observer design originally developed for sampled-data systems in [7] has been extended to NCS in [11]. Assuming a continuous-time observer is known and satisfies some robustness properties with respect to output disturbances, the observation error convergence is ensured under network-induced constraints by replacing the unavailable continuous-time output by an auxiliary variable which flows along the same vector fields between transmission instants and is reset when measurements are received.

In this study, we propose a framework for the observer design for nonlinear NCS via an emulation-like approach, for plants whose dynamics are affected by disturbances. Modeling the problem like in [9], our approach allows to study various types of observers, time-scheduling protocols and in-network processing implementations. We notably recover the designs in [7], [11] as particular cases by showing that it corresponds to a specific choice of in-network processing algorithm. The stability analysis is trajectory-based and carried out using small-gain arguments which allow us to derive easy computable bounds on the maximum allowable transmission interval (MATI). We believe that this is the first time that this problem is addressed with such generality and that our model formulation can be the starting point for other observer designs. An important remark is that our results are also new for the observer design for sampleddata systems. Thus, we provide an alternative to [2] where the emulation of observers for sampled-data systems is investigated based on discrete-time approximate models, noting that we ensure different stability properties here and provide explicit bounds on the maximum allowable sampling period contrary to [2].

The paper is organized as follows. After having defined the notations and recalled some stability definitions in Section II, the problem is stated in Section III and the model formulation is developed. The main results are given in Section IV and applied to a class of globally Lipschitz observers in Section V. 


\section{PRELIMINARIES}

Let $\mathbb{R}=(-\infty, \infty), \mathbb{R}_{\geq 0}=[0, \infty), \mathbb{R}_{>0}=(0, \infty), \mathbb{Z}_{\geq 0}=\{0,1,2, \ldots\}, \mathbb{Z}_{>0}=\{1,2, \ldots\}$. Let $a \in \mathbb{R}_{>0} \cup\{\infty\}$, a function $\gamma:[0, a) \rightarrow \mathbb{R}_{\geq 0}$ is of class $\mathcal{K}$ if it is continuous, zero at zero and strictly increasing. By extension, for $a, b \in \mathbb{R}_{>0} \cup\{\infty\}, \gamma:[0, a) \times[0, b) \rightarrow \mathbb{R}_{\geq 0}$ is of class $\mathcal{K} \mathcal{K}$ if, for any $\left(s_{1}, s_{2}\right) \in[0, a) \times[0, b), \gamma\left(s_{1}, \cdot\right)$ and $\gamma\left(\cdot, s_{2}\right)$ are of class $\mathcal{K}$. A continuous function $\gamma:[0, a) \times \mathbb{R}_{\geq 0} \longrightarrow \mathbb{R}_{\geq 0}$ is of class $\mathcal{K} \mathcal{L}$ if for each $t \in \mathbb{R}_{\geq 0}, \gamma(\cdot, t)$ is of class $\mathcal{K}$, and, for each $s \in[0, a), \gamma(s, \cdot)$ is decreasing to zero. The initial time is denoted $t_{0} \in \mathbb{R}_{\geq 0}$ and the initial condition of a variable $x$ is denoted $x_{0}=x\left(t_{0}\right)$. The Euclidean norm of a vector or a matrix is denoted by $|\cdot|$ and $\lambda_{\min }(\cdot), \lambda_{\max }(\cdot)$ respectively stands for the minimum, maximum eigenvalue of a symmetric positive definite matrix. Let $f: \mathbb{R} \rightarrow \mathbb{R}^{n}, n \in \mathbb{Z}_{>0}$, be a (Lebesgue) measurable function and define, for $t_{1} \leq t_{2} \in \mathbb{R},\|f\|_{\left[t_{1}, t_{2}\right)}=\operatorname{ess}_{\sup } \sup _{\tau \in\left[t_{1}, t_{2}\right)}|f(\tau)|$ and $\|f\|_{\infty}=$ ess. $\sup _{\tau \in\left[t_{0}, \infty\right)}|f(\tau)|$. The set $\mathcal{L}_{\infty}^{n}$ denotes the set of functions $f: \mathbb{R}_{\geq 0} \rightarrow \mathbb{R}^{n}$ such that $\|f\|_{\infty}<r, r \in \mathbb{R}_{\geq 0}$. For $(x, y) \in \mathbb{R}^{n+m}$, the notation $(x, y)$ stands for $\left[x^{\mathrm{T}}, y^{\mathrm{T}}\right]^{\mathrm{T}}$.

Consider the system:

$$
\dot{x}=f(x, u), \quad y=h(x),
$$

where $x \in \mathbb{R}^{n_{x}}, y \in \mathbb{R}^{n_{y}}, u \in \mathbb{R}^{n_{u}}$ are, respectively, the state, the output and the input, $n_{x}, n_{y}, n_{u} \in \mathbb{Z}_{>0}$.

Definition 1: System (1) is input-to-output stable (IOS) from $u$ to $y$ with linear gain $\gamma$ if there exist $\beta \in \mathcal{K} \mathcal{L}$ and $\gamma \in \mathbb{R}_{>0}$ such that, for any $x_{0} \in \mathbb{R}^{n_{x}}, u \in \mathcal{L}_{\infty}^{n_{u}}:|y(t)| \leq \beta\left(\left|x_{0}\right|, t-t_{0}\right)+$ $\gamma\|u\|_{\left[t_{0}, t\right]}$ for $t \geq t_{0} \geq 0$. If $y=x$, then system (1) is input-to-state stable (ISS) w.r.t. $u$.

Definition 2: System (1) is said to be bounded-input-bounded-state (BIBS) with input $u$ if there exist $\alpha, \gamma \in \mathcal{K}$, such that, for any $x_{0} \in \mathbb{R}^{n_{x}}, u \in \mathcal{L}_{\infty}^{n_{u}}:|x(t)| \leq \alpha\left(\left|x_{0}\right|\right)+\gamma\left(\|u\|_{\left[t_{0}, t\right]}\right)$, for $t \geq t_{0} \geq 0$. When no input acts on system (1) (i.e. $u=0$ ), we say that the system is globally stable (GS).

\section{PROBLEM STATEMENT}

The analysis follows the emulation approach adopted for the control of NCS in [15], [9]. The approach consists in first designing the observer while ignoring communication constraints. Thus, for the plant modeled by equations:

$$
\dot{x}=f_{P}(x, w), \quad y=h_{P}(x),
$$


where $x \in \mathbb{R}^{n_{x}}$ is the plant state, $y \in \mathbb{R}^{n_{y}}$ is the plant output, $w \in \mathbb{R}^{n_{w}}$ is an exogenous disturbance input, an observer is synthesized:

$$
\dot{z}=f_{O}(z, y), \quad \bar{x}=h_{O}(z),
$$

where $z \in \mathbb{R}^{n_{z}}$ is the observer state and $\bar{x} \in \mathbb{R}^{n_{x}}$ is the system state estimate. Notice that we allow for immersion-based observers since the dimension of the observer, $n_{z}$, may be bigger than the dimension of the plant, $n_{x}$.

Remark 1: Although in practice, the system output is likely to be corrupted by noise, (2) is disturbance-free here. This model can be justified by the use of a filter whose dynamics are already embedded into (2).

Communication constraints are then taken into account. The variable $y$ is no longer available but $\hat{y}$, which is the generated variable from the most recent system output sent through the network. The problem can be modeled in the following form:

$$
\left.\begin{array}{llr}
\dot{x} & =f_{P}(x, w) & \\
y & =h_{P}(x) & \\
\dot{z} & =f_{O}(z, \hat{y}) & \\
\bar{x} & =h_{O}(z) & \\
\dot{\hat{y}} & =\hat{f}_{P}(\hat{y}, z) & \\
\left.\hat{y}\left(t_{i}^{+}\right)=y\left(t_{i-1}, t_{i}\right]+t_{i-1}\right] \\
\left.t_{i-1}, t_{i}\right]
\end{array}\right\}
$$

where $e=\hat{y}-y \in \mathbb{R}^{n_{e}}\left(n_{e}=n_{y}\right)$ is the network-induced error and the sequence $t_{i}, i \in \mathbb{Z}_{>0}$, of monotonically increasing transmission times satisfies $v \leq t_{i}-t_{i-1} \leq \tau$ for some fixed $\tau \in[v, \infty)$, $t_{0} \in \mathbb{R}_{\geq 0}$ being the initial time ${ }^{1}$. We refer to $\tau$ as the MATI. The network implementation can be described as follows. Grouping sensors into $l$ sensor nodes, where $l \in\left\{1, \ldots, n_{y}\right\}$, the system output is partitioned into $l$ corresponding subvectors, $y=\left(y_{1}, \ldots, y_{l}\right)$. At each transmission instant, exactly one sensor node is chosen to transmit its packet according to the protocol defined by the function $h$. For instance, if the node $k \in\{1, \ldots, l\}$ is selected at time $t_{i}$, we have $\hat{y}_{k}\left(t_{i}^{+}\right)=y_{k}\left(t_{i}\right)$ and $\hat{y}_{j}\left(t_{i}^{+}\right)=\hat{y}_{j}\left(t_{i}\right)$ for $j \neq k$, in other words $e_{k}\left(t_{i}^{+}\right)=0$ and $e_{j}\left(t_{i}^{+}\right)=e_{j}\left(t_{i}\right)$. The function $h$ can be used to model several common protocols in the literature such as round-robin (RR) and try-once-discard protocols (TOD) (see [9] for more details) for instance, or to describe sampled-data systems by setting $h \equiv 0$. The network may also contain

\footnotetext{
${ }^{1}$ Note that $v$ is arbitrary and it is used to prevent Zeno solutions in (4).
} 
an arbitrary number of passive nodes which can only receive packets. They may be used for hosting distant observers and/or actuators for plants with inputs. Without loss of generality, it is assumed that there is only one passive node in the network where an observer is run.

Remark 2: Dynamical protocols such as TOD require the use of smart sensors that have sufficient computational capacities to run a copy of the observer located in the passive node (see Fig. 1 in [3]). At each node $j \in\{1, \ldots, l+1\}$ (where the index $l+1$ denotes the passive node), the observer below is run: $\dot{z}^{j}=f_{O}\left(z^{j}, \hat{y}\right), \bar{x}^{j}=h_{O}\left(z^{j}\right)$. They are synchronized $\left(z^{j}(t)=z^{k}(t)\right.$ for all $t$ and $j, k \in\{1, \ldots, l+1\}$ ) by assuming that they start with the same initial condition and thank to the assignment procedure, which is modeled by a piecewise constant function $\sigma: \mathbb{R}_{\geq 0} \rightarrow\{1, \ldots, l\}$ so that when node $k$ has been assigned at time $t_{i-1}, \sigma(t)=k$ for $t \in\left[t_{i-1}, t_{i}\right]$ and $y_{\sigma\left(t_{i-1}\right)}\left(t_{i-1}\right)=y_{k}\left(t_{i-1}\right)$. In that way, at time $t \in\left[t_{i-1}, t_{i}\right]$, the following signals are available at sensor node $j \in\{1, \ldots, l\}: \sigma\left(t_{i-1}\right), y_{\sigma\left(t_{i-1}\right)}\left(t_{i-1}\right), y_{j}(t), z^{j}(t)$; at the passive node: $\sigma\left(t_{i-1}\right), y_{\sigma\left(t_{i-1}\right)}\left(t_{i-1}\right), z^{l+1}(t)$. Since each observer receives $y_{\sigma\left(t_{i-1}\right)}\left(t_{i-1}\right)$, they all have the same input signal $\hat{y}(t)$ and are synchronised for all time (unless a computational glitch occurs) and the stability analysis reduces to studying a single observer.

Between the transmission instants, $\hat{y}$ is generated according to the in-network processing implementation. Usually zero-order-hold $(\mathrm{ZOH})$ devices are implemented: $\hat{f}_{P}=0$ i.e. the output $\hat{y}$ is kept constant during the transmission intervals. Nevertheless, it has to be noted that more general algorithms can be utilized like the predictive-type used in [7] for sampled-data systems: $\hat{f}_{P}(z)=\frac{\partial h_{P}}{\partial x}\left(h_{O}(z)\right) f_{P}\left(h_{O}(z), 0\right)$, that may help ensuring stronger convergence properties as we will show it in Section V.

Model (4) can be written in a more compact way that is appropriate to our study:

$$
\begin{aligned}
\dot{\xi} & =f_{\xi}(\xi, e, z, w) & \forall t \in\left[t_{i-1}, t_{i}\right] \\
\dot{z} & =f_{z}(\xi, e, z) & \forall t \in\left[t_{i-1}, t_{i}\right] \\
\dot{e} & =g(\xi, e, z, w) & \forall t \in\left[t_{i-1}, t_{i}\right] \\
\xi\left(t_{i}^{+}\right) & =\xi\left(t_{i}\right) & \\
z\left(t_{i}^{+}\right) & =z\left(t_{i}\right) & \\
e\left(t_{i}^{+}\right) & =h\left(i, e\left(t_{j}\right), z\left(t_{i}\right)\right), &
\end{aligned}
$$


where $\xi=x-\bar{x} \in \mathbb{R}^{n_{\xi}}\left(n_{\xi}=n_{x}\right)$ is the observation error and $f_{\xi}(\xi, e, z, w)=f_{P}\left(\xi+h_{O}(z), w\right)-$ $\frac{\partial h_{O}}{\partial z}(z) f_{O}\left(z, e+h_{P}\left(\xi+h_{O}(z)\right)\right), f_{z}(\xi, e, z)=f_{O}\left(z, e+h_{P}\left(\xi+h_{O}(z)\right)\right), g(\xi, e, z, w)=\hat{f}_{P}(e+$ $\left.h_{P}\left(\xi+h_{O}(z)\right), z\right)-\frac{\partial h_{P}}{\partial x}\left(\xi+h_{O}(z)\right) f_{P}\left(\xi+h_{O}(z), w\right)$.

Remark 3: Variables $\xi$ and $z$ could have been embedded into one single vector to be consistent with [9], however we will need to distinguish them in the sequel since we are not interested in the same properties. Indeed, contrary to $\xi$, no convergence property is desired for $z$ but only some well definition or bounded behaviour for all time.

The main problem of this study is to ensure the convergence of the observation error when the plant outputs are transmitted through a serial communication channel.

\section{MAIN RESULTS}

In this section, we propose guidelines for the design of observers for NCS. Assuming the observer (3) ensures some global asymptotic stability properties in the absence of network, we distinguish the cases where the convergence of the observer is maintained practically w.r.t. MATI or asymptotically under communication constraints. We will see in Section V that this difference typically comes from the choice of the in-network processing algorithm.

\section{A. Practical stability}

First, design a continuous-time observer (3) robust to measurement errors in the following sense.

Assumption 1: System (5)-(6) is IOS from $(e, w)$ to $\xi$ with linear gains $\gamma_{1}^{e}, \gamma_{1}^{w}$.

This type of condition was already used for the observer design for sampled-data systems and NCS respectively in [7], [11] and is similar to the IOS assumption for the control of NCS in [9] (condition 2 in Theorem 7).

Second, select a scheduling protocol that satisfies the following property.

Assumption 2: Protocol (10) is uniformly globally exponentially stable (UGES) (see Definition 7 in [9]) with Lyapunov function $W: \mathbb{Z}_{\geq 0} \times \mathbb{R}^{n_{e}} \rightarrow \mathbb{R}_{\geq 0}$ that is locally Lipschitz in $e$ and uniformly in $i$, i.e. there exists $\rho \in[0,1)$ and $\left(a_{1}, a_{2}\right) \in \mathbb{R}_{>0}^{2}$ such that, for all $i \in \mathbb{Z}_{\geq 0},(e, z) \in \mathbb{R}^{n_{e}+n_{z}}$ :

$$
\begin{aligned}
a_{1}|e| & \leq W(i, e) \leq a_{2}|e| \\
W(i+1, h(i, e, z)) & \leq \rho W(i, e) .
\end{aligned}
$$

It has been shown in [9] that the round-robin and the try-once-discard protocols are UGES with locally Lipschitz Lyapunov functions, respectively in view of Propositions 4 and 5 in [9]. The 
sampled-data case also satisfies Assumption 2 with $W(e)=|e|$ for instance, then $a_{1}=a_{2}=1$ and $\rho=0$.

Third, make sure the $e$-system (7) grows exponentially between two successive transmission instants.

Assumption 3: There exist $L, \gamma_{2}^{\xi}, \gamma_{2}^{z}, \gamma_{2}^{w} \in \mathbb{R}_{\geq 0}$, such that, for all $(i, \xi, z, w) \in \mathbb{Z}_{\geq 0} \times \mathbb{R}^{n_{\xi}+n_{z}+n_{w}}$ and almost all $e \in \mathbb{R}^{n_{e}}$ :

$$
\left\langle\frac{\partial W(i, e)}{\partial e}, g(\xi, e, z, w)\right\rangle \leq L W(i, e)+\gamma_{2}^{\xi}|\xi|+\gamma_{2}^{z}|z|+\gamma_{2}^{w}|w| .
$$

This condition is linked to the forward completeness of system (7) in view of [1] and is reminiscent of condition (27) in [9] for the control of NCS.

Fourth, guarantee the boundedness of the states of system (5).

Assumption 4: There exist $\alpha_{3} \in \mathcal{K}$ and $\gamma_{3}^{e}, \gamma_{3}^{w} \in \mathbb{R}_{\geq 0}$ such that, for all $z_{0} \in \mathbb{R}^{n_{z}},(\xi, e, w) \in$ $\mathcal{L}_{\infty}^{n_{\xi}+n_{e}+n_{w}}$, the following holds along solutions to (6):

$$
|z(t)| \leq \alpha_{3}\left(\left|\left(\xi_{0}, z_{0}\right)\right|\right)+\gamma_{3}^{e}\|e\|_{\left[t_{0}, t\right]}+\gamma_{3}^{w}\|w\|_{\left[t_{0}, t\right]} \forall t \geq t_{0} \geq 0
$$

This condition is typically used to guarantee the boundedness of the states of (5)-(10) when using zero-order-hold devices. It is very related to the stability of system (2): we usually prove it by assuming system (2) is BIBS with input $w$ and using Assumption 1 as shown in Section $\mathrm{V}$.

We are now ready to state the main theorem. Its proof is provided in Appendix A. The main idea is to consider system (5)-(10) as the interconnection of three subsystems in $\xi, z$ and $e$ and to apply small-gain arguments to conclude about the stability. Due to the fact that we are dealing with three subsystems (and not two) and that one of them is not expected to converge (z-subsystem (6),(9)), the analysis in [6] had to be modified.

Theorem 1: Under Assumptions 1-4, if $\tau \in\left[v, \tau_{1}\right)$ where $\tau_{1}=\frac{1}{L} \ln \left(\frac{L a_{1}+\gamma_{1}^{e} \gamma_{2}^{\xi}+\gamma_{2}^{z} \gamma_{3}^{e}}{L \rho a_{1}+\gamma_{1}^{e} \gamma_{2}^{\xi}+\gamma_{2}^{z} \gamma_{3}^{e}}\right)\left(\tau_{1}=\right.$ $\frac{a_{1}(1-\rho)}{\gamma_{1}^{e} \gamma_{2}^{\xi}+\gamma_{2}^{z} \gamma_{3}^{e}}$ if $\left.L=0\right)$, then:

- there exist $\beta \in \mathcal{K} \mathcal{L}, \sigma \in \mathcal{K}, \bar{\sigma}, \varepsilon \in \mathcal{K} \mathcal{K}$ such that, for any $\Delta \in \mathbb{R}_{\geq 0},\left(\xi_{0}, e_{0}, z_{0}\right) \in \mathbb{R}^{n_{\xi}+n_{e}+n_{z}}$ with $\left|\left(\xi_{0}, e_{0}, z_{0}\right)\right|<\Delta, w \in \mathcal{L}_{\infty}^{n_{w}}$, this holds for all $t \geq t_{0} \geq 0$

$$
|(\xi(t), e(t))| \leq \beta\left(\left|\left(\xi_{0}, e_{0}, z_{0}\right)\right|, t-t_{0}\right)+\sigma\left(\|w\|_{\infty}\right)+\bar{\sigma}\left(\tau,\|w\|_{\infty}\right)+\varepsilon(\tau, \Delta) .
$$

- system (5)-(10) is BIBS with $w$ as input.

It has to be noticed that (15) is a global property in the sense that the MATI does not depend on the bound on the initial condition $\Delta$. On the other hand, it can be seen that when $w=0$, the 
observation error does not asymptotically converge to the origin but to the ball centered in 0 of radius $\varepsilon(\tau, \Delta)$. This is mainly due to the fact that $\gamma_{2}^{z} \neq 0$ in (13) which typically arises when $\mathrm{ZOH}$ devices are implemented, as shown in Section V. The use of other in-network processing algorithms such as the predictive-type implementation may overcome this issue as they may satisfy slightly different conditions that will ensure the asymptotic convergence to the origin.

\section{B. Asymptotic stability}

We propose an alternative to Section IV-B to guarantee the asymptotic convergence of $(\xi, e)$ to the origin in the absence of disturbances. We slightly strengthen Assumption 1 as follows. Assumption 5: There exist $\beta_{1} \in \mathcal{K} \mathcal{L}, \gamma_{1}^{e}, \gamma_{1}^{w} \in \mathbb{R}_{\geq 0}$ such that, for any $\xi_{0} \in \mathbb{R}^{n_{\xi}},(e, w) \in \mathcal{L}_{\infty}^{n_{e}+n_{w}}$, solutions to (5) satisfy: $|\xi(t)| \leq \beta_{1}\left(\left|\xi_{0}\right|, t-t_{0}\right)+\gamma_{1}^{e}\|e\|_{\left[t_{0}, t\right]}+\gamma_{1}^{w}\|w\|_{\left[t_{0}, t\right]}$ for $t \geq t_{0} \geq 0$.

Remark 4: Assumption 5 implies that system (5) is ISS w.r.t. $(z, e, w)$ with linear gains.

When Assumption 5 holds and Assumption 3 is satisfied with $\gamma_{2}^{z}=0$, the stability of $\xi$ and e-dynamics can be investigated separately from the whole system (5)-(10). In that way, Assumption 4 can be relaxed as follows.

Assumption 6: System (6) is forward complete with input $(\xi, e, w) \in \mathcal{L}_{\infty}^{n_{\xi}+n_{e}+n_{w}}$.

The following theorem can then be derived. Its proof follows similar lines to the proof of Theorem 1, see Appendix B.

Theorem 2: Under Assumptions 2,5,6 and suppose Assumption 3 holds with $\gamma_{2}^{z}=0$. If $\tau \in$ $\left[v, \tau_{2}\right)$ where $\tau_{2}=\frac{1}{L} \ln \left(\frac{L a_{1}+\gamma_{1}^{e} \gamma_{2}^{\xi}}{L \rho a_{1}+\gamma_{1}^{e} \gamma_{2}^{\xi}}\right)\left(\tau_{2}=\frac{a_{1}(1-\rho)}{\gamma_{1}^{e} \gamma_{2}^{\xi}}\right.$ if $\left.L=0\right)$, then:

- there exist $\beta \in \mathcal{K} \mathcal{L}, \sigma \in \mathcal{K}, \bar{\sigma} \in \mathcal{K} \mathcal{K}$ such that, for all $\left(\xi_{0}, z_{0}, e_{0}\right) \in \mathbb{R}^{n_{\xi}+n_{z}+n_{e}}, w \in \mathcal{L}_{\infty}^{n_{w}}$ :

$$
|(\xi(t), e(t))| \leq \beta\left(\left|\left(\xi_{0}, e_{0}\right)\right|, t-t_{0}\right)+\sigma\left(\|w\|_{\infty}\right)+\bar{\sigma}\left(\tau,\|w\|_{\infty}\right) \quad \forall t \geq t_{0} \geq 0
$$

- $\operatorname{system}(5)-(10)$ is forward complete with input $w \in \mathcal{L}_{\infty}^{n_{w}}$.

\section{APPLICATIONS}

In this section, we illustrate the generality of our approach to the observer emulation for NCS. We show that a general class of globally Lipschitz observers which covers the designs in [4], [5], [14] to mention a few, can be implemented over networks ruled by the RR or the TOD protocol, using $\mathrm{ZOH}$ devices or predictive-type implementations and we derive explicit MATI bounds. The case where the system outputs are simply sampled is also considered. 
Consider the system:

$$
\dot{x}=A x+\varrho(x), \quad y=C x,
$$

where $x \in \mathbb{R}^{n_{x}}, y \in \mathbb{R}^{n_{y}}, A$ and $C$ are real matrices of appropriate dimensions and $\varrho: \mathbb{R}^{n_{x}} \rightarrow \mathbb{R}^{n_{x}}$ is globally Lipschitz. The following observer is synthesized:

$$
\dot{\bar{x}}=A \bar{x}+\varrho(\bar{x})+\Lambda(y-\bar{y}), \quad \bar{y}=C \bar{x}
$$

where $\bar{x} \in \mathbb{R}^{n_{x}}, \bar{y} \in \mathbb{R}^{n_{y}}$ and $\Lambda$ is a real matrix, which is of the form of (3) with $z=\bar{x}$. The convergence of the observation error $\xi=x-\bar{x}$ is usually proved using a quadratic Lyapunov function.

Assumption 7: There exists a Lyapunov function $V: \xi \mapsto \xi^{\mathrm{T}} P \xi$ where $P$ is symmetric and positive definite such that: $\frac{\partial V}{\partial \xi}(A x+\varrho(x)-A \bar{x}-\varrho(\bar{x})-\Lambda(y-\bar{y})) \leq-\alpha V$ where $\alpha \in \mathbb{R}_{>0}$.

We now implement observer (18) over a network as described in Section III. Consider the sequence of monotonically increasing transmission instants $t_{i}, i \in \mathbb{Z}_{>0}$, that satisfies $v \leq t_{i}-$ $t_{i-1} \leq \tau$ for all $i \in \mathbb{Z}_{>0}$ and some fixed $v, \tau \in \mathbb{R}_{>0}$. The problem is modeled as:

$$
\begin{array}{rlrl}
\dot{\xi} & =(A-\Lambda C) \xi+\varrho(\xi+z)-\varrho(z)-\Lambda e & & \forall t \in\left[t_{i-1}, t_{i}\right] \\
\dot{z} & =A z+\varrho(z)+\Lambda(e+C \xi) & & \forall t \in\left[t_{i-1}, t_{i}\right] \\
\dot{e} & =\hat{f}_{P}(z)-C A(\xi+z)-C \varrho(\xi+z) & & \forall t \in\left[t_{i-1}, t_{i}\right] \\
\xi\left(t_{i}^{+}\right) & =\xi\left(t_{i}\right) & \\
z\left(t_{i}^{+}\right) & =z\left(t_{i}\right) & \\
e\left(t_{i}^{+}\right) & =h\left(i, e\left(t_{i}\right), z\left(t_{i}\right)\right), &
\end{array}
$$

where $z=\bar{x}, \hat{f}_{P}(z)=0$ or $\hat{f}_{P}(z)=C A z+C \varrho(z)$ whether ZOH devices or the predictive-type algorithm introduced in Section III are implemented. Function $h$ depends whether the protocol is round-robin, try-once-discard (explicit definitions can be found in [9]) or plant outputs are simply sampled $(h \equiv 0)$. We now follow the guidelines of Section IV.

First, we verify that observer (18) is robust to measurement errors as required by Assumptions 1 or 5. Under Assumption 7, we have that along solutions to (19), using the fact that $\lambda_{\min }(P)|\xi|^{2} \leq V(\xi) \leq \lambda_{\max }(P)|\xi|^{2}: \dot{V} \leq-\alpha V-2 \xi^{\mathrm{T}} P \Lambda e \leq-\alpha V+2|\xi||P \Lambda||e| \leq-\alpha V+$ $2 \frac{1}{\sqrt{\lambda_{\min }(P)}} \sqrt{V}|P \Lambda||e|$. Using the formula $2 a b \leq \frac{\alpha}{2} a^{2}+\frac{2}{\alpha} b^{2}$ for $a, b \in \mathbb{R}$, we get: $\dot{V} \leq-\frac{\alpha}{2} V+$ 
$\frac{2}{\alpha} \frac{1}{\lambda_{\min }(P)}|P \Lambda|^{2}|e|^{2}$. Invoking the comparison principle (see Lemma 3.4 in [8]), we have that: $V(t) \leq e^{-\frac{\alpha}{2} t} V(0)+\frac{4}{\alpha^{2}} \frac{1}{\lambda_{\min }(P)}|P \Lambda|^{2}\|e\|_{[0, t]}^{2}$, therefore $|\xi(t)| \leq \beta_{1}\left(\left|\xi_{0}\right|, t\right)+\gamma_{1}^{e}\|e\|_{[0, t]}$ and Assumption 5 (equivalently Assumption 1) is satisfied with $\beta_{1}(s, t)=\sqrt{\frac{\lambda_{\max }(P)}{\lambda_{\min }(P)}} e^{-\frac{\alpha}{4} t} s$ for $(t, s) \in \mathbb{R}_{\geq 0}^{2}$ and $\gamma_{1}^{e}=\frac{2}{\alpha} \frac{1}{\lambda_{\min }(P)}|P \Lambda|$.

Remark 5: It has to be emphasized that we only provide here one possible expression for $\gamma_{1}^{e}$. Others can be determined using different tools, see for instance in Section V in [12] for linear systems or Chapter 5.3.2 in [10] for the observers in [4] (that belong to the studied class of observers).

Second, we have seen in Section IV-A that Assumption 2 holds for RR and TOD protocols and the sampled-data case.

Third, we prove that the $e$-system satisfies the exponential growth condition between transmission instants stated in Assumption 3. The idea is to combine the fact that globally Lipschitz Lyapunov functions are available in [9] for the RR and the TOD protocol (and as a consequence for the sampled-data case) and that the vector field of the $e$-system is globally Lipschitz. We explicitly show it only for the RR protocol, the coefficients for the other network configurations are given in Table I. We consider the following Lyapunov function as done in [9]. For $i \in \mathbb{Z}_{\geq 0}$ and $e \in \mathbb{R}^{n_{e}}, W(i, e)=\sqrt{\sum_{j \in\{1, \ldots, l\}} a_{j}^{2}(i)\left|e_{j}\right|^{2}}=|D(i) e|$, where $D(i)=\operatorname{diag}\left(a_{1}(i) \mathbb{I}_{n_{1}}, \ldots, a_{l}(i) \mathbb{I}_{n_{l}}\right)$ with $a_{j}(i)$ some time-varying coefficients which are such that for any $i \in \mathbb{Z}_{\geq 0}$ and $j \in\{1, \ldots, l\}$ there exists a unique $k \in\{1, \ldots, l\}$ such that $a_{j}^{2}(i)=k$. It can then be deduced that $|D(i)|=\sqrt{l}$, therefore, for $i \in \mathbb{Z}_{\geq 0}$ and $e \in \mathbb{R}^{n_{e}}$ (with $g(\xi, e, z)=\hat{f}_{P}(z)-C A(\xi+z)-C \varrho(\xi+z)$ ),

$$
\left\langle\frac{\partial W(i, e)}{\partial e}, g(\xi, e, z)\right\rangle \leq|D(i) \dot{e}|=\left|D(i)\left(\hat{f}_{P}(z)-C A(\xi+z)-C \varrho(\xi+z)\right)\right|,
$$

this gives for the $\mathrm{ZOH}$ implementation: $\left\langle\frac{\partial W(i, e)}{\partial e}, g(\xi, e, z)\right\rangle \leq \sqrt{l}(|C A|+K)(|\xi|+|z|)$ where $K$ stands for the Lipschitz constant of $C \varrho$. We see that Assumption 3 holds with $L=0$, $\gamma_{2}^{\xi}=\gamma_{2}^{z}=\sqrt{l}(|C A|+K)$. For the predictive-type implementation, i.e. $\hat{f}(z)=C A z+C \varrho(z)$, we obtain: $\left\langle\frac{\partial W(i, e)}{\partial e}, g(\xi, e, z)\right\rangle \leq \sqrt{l}(|C A|+K)|\xi|$, so Assumption 3 is satisfied with $L=0$, $\gamma_{2}^{\xi}=\sqrt{l}(|C A|+K)$ where $\gamma_{2}^{z}=0$.

Fourth, we identify an appropriate sufficient condition on system (17) that ensures that the $z$-system is bounded according to Assumption 4 when using $\mathrm{ZOH}$ devices. Using the fact that $\xi=x-z$, system (20) can be written as: $\dot{z}=(A-\Lambda C) z+\varrho(z)+\Lambda(e+C x)$. As a consequence, 


\begin{tabular}{|c|c|c|}
\cline { 2 - 3 } \multicolumn{1}{c|}{} & ZOH & predictive-type \\
\hline RR protocol & $L=0, \gamma_{2}^{\xi}=\sqrt{l}(|C A|+K), \gamma_{2}^{z}=\sqrt{l}(|C A|+K)$ & $L=0, \gamma_{2}^{\xi}=\sqrt{l}(|C A|+K), \gamma_{2}^{z}=0$ \\
\hline TOD protocol & $L=0, \gamma_{2}^{\xi}=|C A|+K, \gamma_{2}^{z}=|C A|+K$ & $L=0, \gamma_{2}^{\xi}=|C A|+K, \gamma_{2}^{z}=0$ \\
\hline Sampled-data & $L=0, \gamma_{2}^{\xi}=|C A|+K, \gamma_{2}^{z}=|C A|+K$ & $L=0, \gamma_{2}^{\xi}=|C A|+K, \gamma_{2}^{z}=0$ \\
\hline
\end{tabular}

TABLE I

COEFFICIENTS OF ASSUMPTION 3 FOR SYSTEM (21)

by following the same arguments as for proving that (19) is ISS, we get:

$$
|z(t)| \leq \beta_{1}\left(\left|z_{0}\right|, t\right)+\gamma_{1}^{e}\left(\|e\|_{[0, t]}+|C|\|x\|_{[0, t]}\right) .
$$

Now suppose the plant (17) is GS, i.e. there exists $\alpha \in \mathcal{K}$ such that $|x(t)| \leq \alpha\left(\left|x_{0}\right|\right)=\alpha\left(\mid \xi_{0}+\right.$ $\left.z_{0} \mid\right) \leq \alpha\left(2\left|\left(\xi_{0}, z_{0}\right)\right|\right)$. In view of (25), we deduce that: $|z(t)| \leq \beta_{1}\left(\left|z_{0}\right|, t\right)+\gamma_{1}^{e}|C| \alpha\left(2\left|\left(\xi_{0}, z_{0}\right)\right|\right)+$ $\gamma_{1}^{e}\|e\|_{[0, t]} \leq \beta_{1}\left(\left|\left(\xi_{0}, z_{0}\right)\right|, 0\right)+\gamma_{1}^{e}|C| \alpha\left(2\left|\left(\xi_{0}, z_{0}\right)\right|\right)+\gamma_{1}^{e}\|e\|_{[0, t]}$. Thus, Assumption 4 is satisfied with $\gamma_{3}^{e}=\gamma_{1}^{e}$. Note that we do not need to know $\alpha$ to compute $\gamma_{3}^{e}$. We are now ready to apply the results of Section IV. The first proposition is a direct consequence of Theorem 1.

Proposition 1: Consider system (19)-(24) with ZOH in-network processing. Suppose Assumption 7 holds and system (17) is GS. If MATI satisfies $\tau \in\left[v, \tau^{*}\right)$ where $\tau^{*}$ is defined in Table II depending on the protocol, then (15) holds and system (19)-(24) is GS.

Since the nonlinearity of system (20) is globally Lipschitz, Assumption 6 always applies (see Theorem 3.2 in [8]). The following proposition follows from Theorem 2.

Proposition 2: Consider system (19)-(24) with the predictive-type in-network processing and suppose Assumption 7 holds. If MATI satisfies $\tau \in\left[v, \tau^{*}\right)$ where $\tau^{*}$ is defined in Table II depending on the protocol, then (16) holds and system (19)-(24) is forward complete.

\begin{tabular}{|c|c|c|}
\cline { 2 - 3 } \multicolumn{1}{c|}{} & $\mathrm{ZOH}$ & predictive-type \\
\hline RR protocol & $\tau^{*}=\frac{\sqrt{l}-\sqrt{l-1}}{\left.2 l \gamma_{1}^{e}|C A|+K\right)}$ & $\tau^{*}=\frac{\sqrt{l}-\sqrt{l-1}}{l \gamma_{1}^{e}(|C A|+K)}$ \\
\hline TOD protocol & $\tau^{*}=\frac{\sqrt{l}-\sqrt{l-1}}{2 \sqrt{l} \gamma_{1}^{e}(|C A|+K)}$ & $\tau^{*}=\frac{\sqrt{l}-\sqrt{l-1}}{\sqrt{l} \gamma_{1}^{e}(|C A|+K)}$ \\
\hline Sampled-data & $\tau^{*}=\frac{1}{2 \gamma_{1}^{e}(|C A|+K)}$ & $\tau^{*}=\frac{1}{\gamma_{1}^{e}(|C A|+K)}$ \\
\hline
\end{tabular}

TABLE II

MATI BOUNDS FOR SYSTEM (19)-(24) 


\section{CONCLUSION}

We have developed a framework for the observer design for NCS affected by disturbances, via an emulation-based approach. The proposed model allows us to study various observers, time-scheduling protocols and in-network processing implementations. Sufficient conditions on the system are given and explicit MATI bounds are deduced which ensure the convergence of the observation error under network-induced communication constraints. The extension of this study to the case where the ISS / IOS assumptions hold with nonlinear gains and the protocol is uniformly globally asymptotically stable is available in [13]. A consequence is that the obtained stability properties are no longer global but semiglobal and practical in general and the computation of the MATI bounds becomes more involved.

\section{REFERENCES}

[1] D. Angeli and E.D. Sontag. Forward completeness, unboundedness observability, and their Lyapunov characterizations. Systems \& Control Letters, 38:209-217, 1999.

[2] M. Arcak and D. Nešić. A framework for nonlinear sampled-data observer design via approximate discrete-time models and emulation. Automatica, 40:1931-1938, 2004.

[3] D. Dačić and D. Nešić. Observer design for linear networked control systems using matrix inequalities. Automatica, 44(1):2840-2848, 2008.

[4] M. Farza, M. M’Saad, and L. Rossignol. Observer design for a class of MIMO nonlinear systems. Automatica, 40:135-143, 2004.

[5] J.P. Gauthier, H. Hammouri, and S. Othman. A simple observer for nonlinear systems - applications to bioreactors. IEEE Transactions on Automatic Control, 37:875-880, 1992.

[6] Z.-P. Jiang, A.R. Teel, and L. Praly. Small-gain theorem for ISS systems and applications. Mathematics of Control, Signals, and Systems, 7:95-120, 1994.

[7] I. Karafyllis and C. Kravaris. From continuous-time design to sampled-data design of observers. IEEE Transactions on Automatic Control, 54(9):2169-2174, 2009.

[8] H.K. Khalil. Nonlinear Systems. Prentice-Hall, Englewood Cliffs, New Jersey, U.S.A., 3rd edition, 2002.

[9] D. Nešić and A.R. Teel. Input-output stability properties of networked control systems. IEEE Transactions on Automatic Control, 49:1650-1667, 2004.

[10] R. Postoyan. Commande et construction d'observateurs pour les systèmes non linéaires à données échantillonnées et en réseau. PhD thesis, Univ Paris-Sud (in French), 2009.

[11] R. Postoyan, T. Ahmed-Ali, and F. Lamnabhi-Lagarrigue. Observers for classes of nonlinear networked systems. In SSD’09 (IEEE International Conference on Systems, Signals and Devices), Djerba, Tunisia, 2009.

[12] R. Postoyan and D. Nešić. A framework for the observer design for networked control systems. In ACC (American Control Conference), Baltimore, U.S.A., pages 3678-3683, 2010.

[13] R. Postoyan and D. Nešić. On emulation-based observer design for networked control systems. In CDC (IEEE Conference on Decision and Control) Atlanta, U.S.A., 2010.

[14] R. Rajamani. Observers for Lipschitz nonlinear systems. IEEE Transactions on Automatic Control, 43(3):397-401, 1998. 
[15] G.C. Walsh, O. Beldiman, and L.G. Bushnell. Asymptotic behavior of nonlinear networked control systems. IEEE Transactions on Automatic Control, 46:1093-1097, 2001.

[16] L. Zhang and D. Hristu-Varsakelis. Stabilization of networked control system: designing effective communication sequence. In $16^{\text {th }}$ IFAC World Congress, Prague, Czech Republic, 2005.

\section{APPENDIX A}

\section{PROOF OF THEOREM 1}

The proof follows the main lines of the proof of Theorem 2.1 in [6] under appropriate modifications due to the fact that we consider the interconnection of three systems (and not two) and that the $z$-system (6),(9) exhibits a boundedness property and not an ISS / IOS property. Step 1: BIBS property. Let $\left(\xi_{0}, e_{0}, z_{0}\right) \in \mathbb{R}^{n_{\xi}+n_{e}+n_{z}}, w \in \mathcal{L}_{\infty}^{n_{w}}$ and $\tau \in\left[v, \tau^{*}\right)$. Let $\left[t_{0}, t_{\max }\right)$ denote the maximum existence interval for system (5)-(10), where $t_{\max } \in\left(t_{0}, \infty\right]$. Let $t \in$ $\left[t_{0}, t_{\max }\right)$. Since Assumptions 2-3 hold, according to Proposition 6 in [9] and using the fact that $a_{1}|e| \leq W(e)$ from (11), there exists $\beta_{2} \in \mathcal{K} \mathcal{L}$ such that:

$$
|e(t)| \leq \beta_{2}\left(\left|e_{0}\right|, t-t_{0}\right)+\zeta(\tau)\left(\gamma_{2}^{\xi}\|\xi\|_{\left[t_{0}, t\right]}+\gamma_{2}^{z}\|z\|_{\left[t_{0}, t\right]}+\gamma_{2}^{w}\|w\|_{\left[t_{0}, t\right]}\right),
$$

where $\zeta(\tau)=\frac{\exp (L \tau)-1}{a_{1} L(1-\rho \exp (L \tau))}$ (if $L=0, \zeta(\tau)=\frac{\tau}{a_{1}(1-\rho)}$ ) that defines a class- $\mathcal{K}$ function on $\left[0, \tau^{*}\right.$ ) (it can be noted that $\tau^{*}<\frac{1}{L} \ln \rho^{-1}$ so that $\zeta$ is well defined). On the other hand, in view of Assumption 1, there exists $\beta_{1} \in \mathcal{K} \mathcal{L}$ such that:

$$
|\xi(t)| \leq \beta_{1}\left(\left|\left(\xi_{0}, z_{0}\right)\right|, t-t_{0}\right)+\gamma_{1}^{e}\|e\|_{\left[t_{0}, t\right]}+\gamma_{1}^{w}\|w\|_{\left[t_{0}, t\right]} .
$$

Thus, in view of (26), (27) and Assumption 4, we have that:

$$
\|e\|_{\left[t_{0}, t\right]} \leq \beta_{2}\left(\left|e_{0}\right|, 0\right)+\zeta(\tau)\left(\gamma_{2}^{\xi}\|\xi\|_{\left[t_{0}, t\right]}+\gamma_{2}^{z}\|z\|_{\left[t_{0}, t\right]}+\gamma_{2}^{w}\|w\|_{\left[t_{0}, t\right]}\right) \leq M_{e}\left(\tau, \xi_{0}, e_{0}, z_{0},\|w\|_{\left[t_{0}, t\right]}\right),
$$

where $M_{e}\left(\tau, \xi_{0}, e_{0}, z_{0},\|w\|_{\left[t_{0}, t\right]}\right)=\frac{1}{d(\tau)} \beta_{2}\left(\left|e_{0}\right|, 0\right)+\frac{\zeta(\tau)}{d(\tau)}\left(\gamma_{2}^{\xi} \beta_{1}\left(\left|\left(\xi_{0}, z_{0}\right)\right|, 0\right)+\gamma_{2}^{z} \alpha_{3}\left(\left|\left(\xi_{0}, z_{0}\right)\right|\right)+\right.$ $\left.\left(\gamma_{2}^{\xi} \gamma_{1}^{w}+\gamma_{2}^{z} \gamma_{3}^{w}+\gamma_{2}^{w}\right)\|w\|_{[0, t]}\right)$ and $d(\tau)=1-\zeta(\tau)\left(\gamma_{2}^{\xi} \gamma_{1}^{e}+\gamma_{2}^{z} \gamma_{3}^{e}\right)$ that satisfies $d(\tau)>0$ since $\tau \in\left[v, \tau^{*}\right)$. It can then be shown that:

$$
|\xi(t)| \leq M_{\xi}\left(\tau, \xi_{0}, e_{0}, z_{0},\|w\|_{\left[t_{0}, t\right]}\right), \quad|z(t)| \leq M_{z}\left(\tau, \xi_{0}, e_{0}, z_{0},\|w\|_{\left[t_{0}, t\right]}\right),
$$

with (for the purpose of clarity, we omit the arguments of functions $M_{\xi}$ and $M_{z}$ ): $M_{\xi}=$ $\beta_{1}\left(\left|\left(\xi_{0}, z_{0}\right)\right|, 0\right)+\gamma_{1}^{e} M_{e}+\gamma_{1}^{w}\|w\|_{\left[t_{0}, t\right]}, M_{z}=\alpha_{3}\left(\left|\left(\xi_{0}, z_{0}\right)\right|\right)+\gamma_{3}^{e} M_{e}+\gamma_{3}^{w}\|w\|_{\left[t_{0}, t\right]}$. It follows that,

$$
|(\xi(t), e(t), z(t))| \leq M_{\xi}+M_{e}+M_{z}=\alpha\left(\left|\left(\xi_{0}, e_{0}, z_{0}\right)\right|\right)+\vartheta\|w\|_{\left[t_{0}, t\right]},
$$


where $\alpha \in \mathcal{K}$ and $\vartheta \in \mathbb{R}_{\geq 0}$. Therefore, system (5)-(10) is BIBS in view of Definition 2 and $t_{\max }=\infty$.

Step 2: Convergence property. For any $t_{0} \leq t_{10} \leq t_{20} \leq t_{11} \leq t_{21}$, using time invariance and causality of the inequalities (26) and (27):

$$
\begin{aligned}
&\left.\mid \xi\left(t_{11}\right)\right) \mid \leq \beta_{1}\left(\left|\left(\xi\left(t_{10}\right), z\left(t_{10}\right)\right)\right|, t_{11}-t_{10}\right)+\gamma_{1}^{e}\|e\|_{\left[t_{10}, t_{11}\right]}+\gamma_{1}^{w}\|w\|_{\infty} \\
&\left|e\left(t_{21}\right)\right| \leq \beta_{2}\left(\left|e\left(t_{20}\right)\right|, t_{21}-t_{20}\right)+\zeta(\tau)\left(\gamma_{2}^{\xi}\|\xi\|_{\left[t_{20}, t_{21}\right]}+\gamma_{2}^{z}\|z\|_{\infty}+\gamma_{2}^{w}\|w\|_{\infty}\right) .
\end{aligned}
$$

Let $t \in\left[t_{0}, \infty\right)$, taking $t_{10}=\frac{t-t_{0}}{4}+t_{0}, t_{20}=\frac{t-t_{0}}{2}+t_{0}, t_{21}=t, t_{11} \in\left[\frac{t-t_{0}}{2}+t_{0}, t\right]$, in view of (26), (28), (29), (29) and (31):

$$
\begin{aligned}
|e(t)| \leq & \beta_{2}\left(M_{e}, \frac{t-t_{0}}{2}\right)+\zeta(\tau)\left(\gamma_{2}^{\xi} \beta_{1}\left(M_{\xi}+M_{z}, \frac{t-t_{0}}{4}\right)+\gamma_{2}^{\xi} \gamma_{1}^{e}\|e\|_{\left[\frac{t-t_{0}}{4}+t_{0}, \infty\right)}\right. \\
& \left.+\gamma_{2}^{z}\|z\|_{\infty}+\left(\gamma_{2}^{\xi} \gamma_{1}^{w}+\gamma_{2}^{w}\right)\|w\|_{\infty}\right) \\
\leq & \beta_{2}\left(M_{e}, \frac{t-t_{0}}{2}\right)+\zeta\left(\tau^{*}\right) \gamma_{2}^{\xi} \beta_{1}\left(M_{\xi}+M_{z}, \frac{t-t_{0}}{4}\right) \\
& +\zeta(\tau)\left(\gamma_{2}^{\xi} \gamma_{1}^{e}\|e\|_{\left[\frac{t-t_{0}}{4}+t_{0}, \infty\right)}+\gamma_{2}^{z}\|z\|_{\infty}+\left(\gamma_{2}^{\xi} \gamma_{1}^{w}+\gamma_{2}^{w}\right)\|w\|_{\infty}\right) .
\end{aligned}
$$

Considering (34) and noticing that $\zeta(\tau) \gamma_{2}^{\xi} \gamma_{1}^{e}<1$ (since $\tau<\tau^{*}$ ), Lemma A.1 in [6] is used to show (with $z(t)=|e(t)|, \beta(s, t)=\beta_{2}\left(s, \frac{t}{2}\right)+\zeta\left(\tau^{*}\right) \gamma_{2}^{\xi} \beta_{1}\left(s, \frac{t}{4}\right), s=M_{e}+M_{\xi}+M_{z}, \rho(s)=$ $\zeta(\tau) \gamma_{2}^{\xi} \gamma_{1}^{e} s, d=\zeta(\tau)\left(\gamma_{2}^{z}\|z\|_{\infty}+\left(\gamma_{2}^{\xi} \gamma_{1}^{w}+\gamma_{2}^{w}\right)\|w\|_{\infty}\right)$ and $\left.\mu=\frac{1}{4},(s, t) \in \mathbb{R}_{\geq 0}^{2}\right)$ that there exist $\lambda_{2} \in(1, \infty)$ and $\tilde{\beta}_{2} \in \mathcal{K} \mathcal{L}$ such that:

$$
|e(t)| \leq \tilde{\beta}_{2}\left(M_{e}+M_{\xi}+M_{z}, t-t_{0}\right)+\frac{\lambda_{2} \zeta(\tau)}{1-\zeta(\tau) \gamma_{2}^{\xi} \gamma_{1}^{e}}\left(\gamma_{2}^{z}\|z\|_{\infty}+\left(\gamma_{2}^{\xi} \gamma_{1}^{w}+\gamma_{2}^{w}\right)\|w\|_{\infty}\right) .
$$

Similarly, in view of (31), it can be shown that there exist $\tilde{\beta}_{1} \in \mathcal{K} \mathcal{L}$ and $\lambda_{1} \in(1, \infty)$ such that,

$$
|\xi(t)| \leq \tilde{\beta}_{1}\left(M_{e}+M_{\xi}+M_{z}, t-t_{0}\right)+\frac{\lambda_{1}}{1-\zeta(\tau) \gamma_{2}^{\xi} \gamma_{1}^{e}}\left(\zeta(\tau) \gamma_{1}^{e} \gamma_{2}^{z}\|z\|_{\infty}+\left(\gamma_{1}^{w}+\zeta(\tau) \gamma_{1}^{e} \gamma_{2}^{w}\right)\|w\|_{\infty}\right) .
$$

By combining (35) and (36) and introducing $\tilde{\beta}=\tilde{\beta}_{1}+\tilde{\beta}_{2}$, it follows that:

$$
\begin{aligned}
|(\xi(t), e(t))| \leq & \tilde{\beta}\left(M_{e}+M_{\xi}+M_{z}, t-t_{0}\right)+\frac{\zeta(\tau)}{1-\zeta(\tau) \gamma_{2}^{\xi} \gamma_{1}^{e}}\left(\lambda_{1} \gamma_{1}^{e} \gamma_{2}^{z}+\lambda_{2} \gamma_{2}^{z}\right)\|z\|_{\infty} \\
& +\frac{1}{1-\zeta(\tau) \gamma_{2}^{\xi} \gamma_{1}^{e}}\left(\lambda_{1}\left(\gamma_{1}^{w}+\zeta(\tau) \gamma_{1}^{e} \gamma_{2}^{w}\right)+\lambda_{2} \zeta(\tau)\left(\gamma_{2}^{\xi} \gamma_{1}^{w}+\gamma_{2}^{w}\right)\right)\|w\|_{\infty}
\end{aligned}
$$

According to (30) and using the fact that $\tilde{\beta}(a+b, t) \leq \tilde{\beta}(2 a, t)+\tilde{\beta}(2 b, t)$ for any $a, b, t \in \mathbb{R}_{\geq 0}$ since $\tilde{\beta}(\cdot, t) \in \mathcal{K}$, we have that: $\tilde{\beta}\left(M_{e}+M_{\xi}+M_{z}, t-t_{0}\right)=\tilde{\beta}\left(\alpha\left(\left|\left(\xi_{0}, e_{0}, z_{0}\right)\right|\right)+\vartheta\|w\|_{\left[t_{0}, t\right]}, t-t_{0}\right) \leq$ $\tilde{\beta}\left(2 \alpha\left(\left|\left(\xi_{0}, e_{0}, z_{0}\right)\right|\right), t-t_{0}\right)+\tilde{\beta}\left(2 \vartheta\|w\|_{\left[t_{0}, t\right]}, 0\right)$, consequently, in view of (30) and (37), taking any $\Delta \in \mathbb{R}_{\geq 0}$ and $\left(\xi_{0}, e_{0}, z_{0}\right) \in \mathbb{R}^{n_{\xi}+n_{e}+n_{z}}$ with $\left|\left(\xi_{0}, e_{0}, z_{0}\right)\right|<\Delta, t \in\left[t_{0}, \infty\right)$ we have that: 


$$
|(\xi(t), e(t))| \leq \beta\left(\left|\left(\xi_{0}, e_{0}, z_{0}\right)\right|, t-t_{0}\right)+\sigma\left(\|w\|_{\infty}\right)+\varsigma\left(\tau,\|w\|_{\infty}\right)+\varepsilon(\tau, \Delta),
$$

where, noting that $\frac{1}{1-\zeta(\tau) \gamma_{2}^{\xi} \gamma_{1}^{e}}=1+\frac{\zeta(\tau) \gamma_{2}^{\xi} \gamma_{1}^{e}}{1-\zeta(\tau) \gamma_{2}^{\xi} \gamma_{1}^{e}}$, for $(s, t) \in \mathbb{R}_{\geq 0}^{2}, \beta(s, t)=\tilde{\beta}(2 \alpha(s), t), \sigma(s)=$ $\tilde{\beta}(2 \vartheta s, 0)+\lambda_{1} \gamma_{1}^{w} s, \varsigma(\tau, s)=\frac{\zeta(\tau)}{1-\zeta(\tau) \gamma_{2}^{\xi} \gamma_{1}^{e}}\left(\lambda_{1} \gamma_{1}^{e}+\lambda_{2}\right) \gamma_{2}^{z} \vartheta s+\frac{\zeta(\tau) \gamma_{2}^{\xi} \gamma_{1}^{e}}{1-\zeta(\tau) \gamma_{2}^{\xi} \gamma_{1}^{e}} \lambda_{1} \gamma_{1}^{w} s+\frac{\zeta(\tau)}{1-\zeta(\tau) \gamma_{2}^{\xi} \gamma_{1}^{e}}\left(\lambda_{1} \gamma_{1}^{e} \gamma_{2}^{w}+\right.$ $\left.\lambda_{2}\left(\gamma_{2}^{\xi} \gamma_{1}^{w}+\gamma_{2}^{w}\right)\right) s, \varepsilon(\tau, s)=\frac{\zeta(\tau)}{1-\zeta(\tau) \gamma_{2}^{\xi} \gamma_{1}^{e}}\left(\lambda_{1} \gamma_{1}^{e}+\lambda_{2}\right) \gamma_{2}^{z} \alpha(3 s)$. We have that $\beta \in \mathcal{K} \mathcal{L}$ and $\sigma, \varsigma(\tau, \cdot)$, $\varepsilon(\tau, \cdot) \in \mathcal{K}$. Now take $s \in \mathbb{R}_{>0}$, it can noticed that $\varsigma(\cdot, s), \varepsilon(\cdot, s)$ are continuous on $\left[0, \tau^{*}\right)$ and equal to 0 at 0 (since $\zeta$ is continuous on $\left[0, \tau^{*}\right)$ and $\zeta(0)=0$ ). Furthermore, since $\zeta$ is strictly increasing on $\left[0, \tau^{*}\right)$, so is $\tau \mapsto \frac{\zeta(\tau)}{1-\zeta(\tau) \gamma_{2}^{\xi} \gamma_{1}^{e}}$, we have that $\varsigma(\cdot, s), \varepsilon(\cdot, s) \in \mathcal{K}$. The desired result (15) is obtained.

\section{APPENDIX B}

\section{SKETCH OF PROOF OF THEOREM 2}

Using the forward completeness characterization given in Corollary 2.3 in [1], we have that Assumption 6 is equivalent to the following. There exist $\nu_{1}, \nu_{2}, \nu_{3} \in \mathcal{K}$ and $c \in \mathbb{R}_{\geq 0}$ such that, for any $z_{0} \in \mathbb{R}^{n_{z}},(\xi, e, w) \in \mathcal{L}_{\infty}^{n_{e}+n_{\xi}+n_{w}}$, along solutions to $(6):|z(t)| \leq \nu_{1}(t)+\nu_{2}\left(\left|z_{0}\right|\right)+$ $\nu_{3}\left(\|(\xi, e, w)\|_{\left[t_{0}, t\right]}\right)+c$ for all $t \geq t_{0} \geq 0$. The proof then immediately follows from the proof of Theorem 1 by making $\beta_{1}$ only depends on $\left|\xi_{0}\right|$ (and not $\left|\left(\xi_{0}, z_{0}\right)\right|$ ) and setting $\gamma_{2}^{z}=0$. We then obtain that $\varepsilon$ is equal to 0 and appropriate $\sigma$ and $\varsigma$ can be deduced. 